\title{
Comparação entre Técnicas de Fotogrametria e Escaneamento de Luz Estruturada para Reconstrução de Objetos em 3D
}

\author{
Comparison between Photogrammetry and Structured Light Scanning Techniques for 3D
}

\author{
Object Reconstruction
}

\author{
Júlia Tannús*, Isabela Favareto ${ }^{\dagger}$, Edgard Lamounier ${ }^{\ddagger}$, Alexandre Cardoso ${ }^{\S}$ \\ Faculdade de Engenharia Elétrica \\ Universidade Federal de Uberlândia \\ Uberlândia, Brasil \\ *julia.tannus95@gmail.com, ${ }^{\dagger}$ isabelafavareto@gmail.com, ${ }^{\ddagger}$ lamounier@ufu.br, ${ }^{\S}$ alexandre@ufu.br
}

\begin{abstract}
Resumo-Modelos 3D de alto realismo possuem muitas possibilidades de aplicação em diversas áreas. Porém, a modelagem 3D manual demanda tempo e habilidade. Por isto, técnicas de reprodução tridimensional automática estão sendo estudadas, tornando-se cada vez mais avançadas e disponíveis para diversos cenários e aplicações. Entre elas, destacam-se o escaneamento 3D e a fotogrametria computadorizada. Contudo, ainda não há um consenso acerca de qual a melhor técnica a ser utilizada para a geração de modelos tridimensionais. Neste artigo, foi feito um modelo 3D a partir de um objeto real usando cada uma das técnicas, a fim de compará-las entre si e com o próprio com base em três componentes: precisão geométrica de cada modelo com relação ao objeto real, precisão relativa entre os dois produtos gerados e análise visual qualitativa. Observou-se que o modelo escaneado apresentou maior diferença de distâncias e pior qualidade em relação ao obtido por fotogrametria, apesar deste último demandar maior tempo de processamento.
\end{abstract}

Palavras-chave-Escaneamento 3D, Fotogrametria, Realidade Virtual

Abstract-High realism 3D models have many possibilities of application in several areas. However, manual 3D modeling takes time and skill. Therefore, three-dimensional automatic reproduction techniques are being studied, becoming increasingly advanced and available for various scenarios and applications. Among them, 3D scanning and computerized photogrammetry stand out. However, there is still no consensus as to which technique is best used for the generation of three-dimensional models. In this article, a 3D model was made from a real object using each of the techniques, in order to compare them with each other and with itself based on three components: geometric precision of each model with respect to the real object, relative precision between the two products generated and qualitative visual analysis. It was observed that the scanned model presented greater difference of distances and worse quality in relation to the one obtained by photogrammetry, despite the latter requiring a longer processing time.

Index Terms-3D Scanning, Photogrammetry, Virtual Reality

\section{INTRODUÇÃO}

A Computação Gráfica, desde seus primórdios, busca reproduzir com fidelidade o mundo real. Modelos 3D de alto realismo possuem muitas possibilidades de aplicação, por exemplo, na arqueologia, preservando peças de museus antes da sua degradação, podendo compartilhar com várias pessoas ao redor do mundo por meio da Internet, rompendo as barreiras físicas [1]. Outras possibilidades podem ser citadas nos campos da anatomia, agricultura, comércio e jogos virtuais [2], [3].

Por este motivo, técnicas automáticas de fabricação de modelos 3D têm sido exploradas, já que a modelagem 3D tradicional demanda tempo, habilidade e trabalho manual. Dentre as técnicas atuais, destacam-se o escaneamento 3D e a fotogrametria computadorizada.

De acordo com a American Society of Photogrammetry, a fotogrametria pode ser definida como: "a arte, ciência e tecnologia de obtenção de informação confiável sobre objetos físicos e o meio ambiente por meio de processos de gravação, medição e interpretação de imagens fotográficas e padrões de energia eletromagnética radiante e outras fontes." De fato, a fotogrametria é a técnica de reconstituição tridimensional criada a partir de imagens bidimensionais [1].

Já o escaneamento 3D é um método de varredura de alta acurácia e precisão que permite a captura automática de grande quantidade de dados em curto espaço de tempo [4]. Esse recurso baseia-se na análise e medição tridimensional, podendo utilizar scanners a laser ou de luz estruturada, sensores de profundidade e aplicativos de celular para a geração de um modelo digital de um objeto.

As duas técnicas de reprodução tridimensional estão sendo cada vez mais utilizadas e inseridas no mercado [5], possuindo uma vasta área de aplicações, desde a utilização para a elaboração de próteses médicas, até o registro e documentação de artefatos e construções [6], [7]. Ambos os métodos possuem algumas diferenças entre si, tais quais o nível de precisão, a resolução do modelo 3D produzido, o custo do equipamento e o tempo que leva para ser realizado cada procedimento [5]. Contudo, ainda não se tem uma conclusão acerca de qual a melhor técnica a ser utilizada para a geração de modelos tridimensionais [8].

Em vista disso, este artigo apresenta uma comparação entre a fotogrametria computadorizada e o escaneamento $3 \mathrm{D}$, em 
específico com o scanner Structure Sensor [9], a fim de verificar a eficácia de cada método na reprodução de modelos reais em virtuais e concluir qual o mais adequado para cada aplicação. Considerou-se justo o confronto pela similaridade de preços entre os dispositivos utilizados para as duas técnicas (scanner e câmera).

\section{Trabalhos Correlatos}

Esta seção apresenta alguns trabalhos na mesma área de pesquisa de comparação de diferentes métodos utilizados para a geração de modelos 3D.

No trabalho em [8], são testados objetos de diferentes escalas e texturas para fotogrametria baseada em imagens e um scanner a laser. Foi testada a geração de modelos tridimensionais de uma esfera texturizada de $300 \mathrm{~mm}$, a fachada de um edifício plano e uma rede elétrica bem complexa. Os resultados do trabalho mostraram que para objetos de pequenos e médios tamanhos e distâncias, a qualidade da fotogrametria combinada com o software se sobrepõe ao scanner. Já para objetos de grande escala, o scanner produziu modelos de maior qualidade.

Para o trabalho em [10], foi feita a comparação dos erros e distorções entre a fotogrametria digital e o scanner a laser, onde diferentes objetos foram testados sob diferentes configurações, a fim de obter o melhor desempenho e precisão do scanner. Os resultados mostraram que o scanner apresentou maiores distorções que a fotogrametria digital, estas milimétricas, e, apresentou ainda maior dificuldade em obter maior precisão na parte inferior do objeto do que na superior, erros que podem ser justificados pelo ruído de medição do scanner e dificuldade de interpolação do modelo.

Já no trabalho em [11], comparações de alguns critérios, tais quais a qualidade das imagens, o tempo e custo necessários, e os problemas encontrados nos processos, foram feitos com o intuito de constatar qual o melhor método para cada situação e objeto escolhido, seja a fotogrametria, o escaneamento 3D, ou até uma associação dos dois. Variados objetos foram escolhidos como alvo do estudo, como estátuas antigas e pedras arqueológicas. Os resultados apontaram que a fotogrametria é preferível na maioria dos casos, principalmente para o caso de objetos com texturas distintas. Além disso, o trabalho pontuou o pouco tempo que é necessário para a realização das fotos. Já para o caso de objetos complexos e irregulares, é preferível a utilização do escaneamento 3D, que confere apresentações muito precisas e realistas.

Por meio destes estudos, pôde-se notar que o melhor método a ser escolhido dependerá muito do objeto a ser reproduzido, de como os processos foram realizados e onde os modelos serão utilizados. Contudo, majoritariamente, a fotogrametria foi eleita e empregada como melhor recurso para a obtenção das imagens com maior qualidade e detalhamento dos objetos.

Além disso, foi possível observar que nenhum trabalho apresentou a comparação aqui proposta, ou seja, o confronto entre o escaneamento 3D utilizando um scanner de luz estruturada e a fotogrametria para geração de objetos para ambientes virtuais.

\section{Materiais e Métodos}

Para produzir os modelos 3D, em primeiro lugar, foi escolhido um objeto que apresentasse alguma dificuldade específica a ser aferida e um local propício para captura das imagens. O objeto eleito foi um baú de madeira, adorno doméstico, cuja aparência e dimensões aproximadas são apresentadas na Figura 1

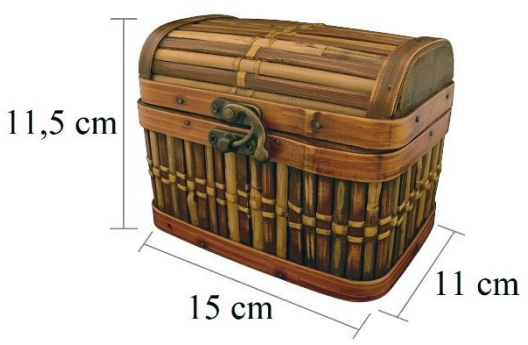

Figura 1. Foto do objeto real e suas medidas aproximadas.

O baú foi escolhido por apresentar planos retos e o formato similar ao de um paralelepípedo. A comparação com esta forma primitiva possibilita boa visualização de eventuais distorções na forma do modelo virtual. Também apresenta ângulos curvos e saliências de tamanhos diversos, tamanho diminuto e superfícies difusas (não refletoras).

O local eleito para captura de fotos e escaneamento 3D foi ao ar livre, num dia ensolarado, sob a penumbra de uma árvore (a luz solar direta acarretaria em sombras e reflexos indesejados no modelo final). $\mathrm{O}$ objeto foi posto em cima de uma banqueta. Tanto as fotos quanto o escaneamento foram feitos a aproximadamente $60 \mathrm{~cm}$ do objeto, dando 2 voltas ao redor dele, sendo uma das voltas aproximadamente no plano horizontal e outra aproximadamente a $45^{\circ}$ do mesmo, segundo esquema da Figura 2 Não foram usados dispositivos auxiliares (por exemplo, tripé) além da câmera e do scanner.

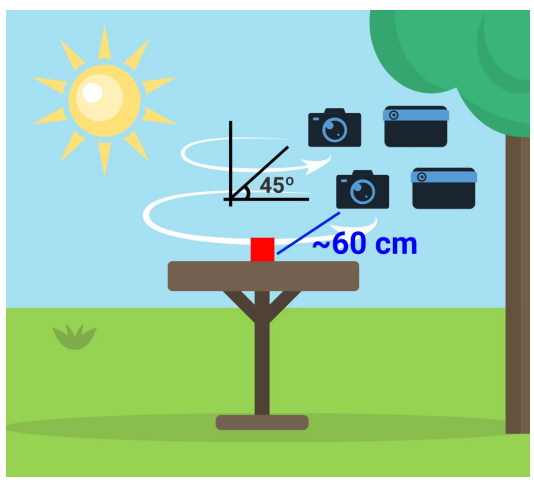

Figura 2. Esquema da captura de fotos e escaneamento 3D.

Antes de fazer o escaneamento, foi feita a calibração do aparelho por meio do aplicativo para iPad "Structure Sensor Calibrator". Em seguida, foi aberto o aplicativo "Scanner Structure SDK" (ambos fornecidos pelo fabricante do sensor) e feito o escaneamento conforme instruções do aparelho, utilizando as configurações de máxima qualidade. $\mathrm{O}$ aplicativo 
"Scanner" é de simples utilização, basta apontar o sensor para o objeto, que um cubo transparente deve aparecer na tela. Por meio de toques, ajusta-se o tamanho do cubo para corresponder ao volume que se deseja escanear. Após isto, basta apertar o botão "Scan" e lentamente mover-se ao redor do objeto, a fim de completar todas as lacunas do modelo. Finalmente, exporta-se o modelo no formato .OBJ.

Já para a captura de fotos para fotogrametria computadorizada foi utilizada uma câmera DSLR de 24,2 MP, e nela aplicadas as seguintes configurações: dimensão das fotos 6016x4000 pixels, resolução $300 \mathrm{dpi}$, abertura f/8, tempo de exposição 1/125 s, ISO-200, distância focal $18 \mathrm{~mm}$ e flash desativado. As fotos não receberam nenhum tipo de tratamento posterior.

A seguir, as fotos foram processadas em um notebook com processador Intel i7 ( $7^{\text {a }}$ geração), memória $8 \mathrm{~GB}$, placa de vídeo Geforce 940MX com 2GB de memória, no software Agisoft Photoscan [12]. Este software usa imagens de objetos estacionários para gerar nuvens de pontos e modelos 3D (método "Structure from Motion"). É amplamente utilizado em fotogrametria de aviação e engenharia, animações por computador e sensoriamento remoto [13]. Com a capacidade de exportar dados processados para uma variedade de formatos, é compatível com diversos programas gráficos. As fotos foram alinhadas e a nuvem de pontos densa construída na configuração de qualidade média e o modelo foi composto na qualidade baixa.

A avaliação dos resultados finais se concentrou em três componentes principais: precisão geométrica de cada modelo com relação ao objeto real, precisão relativa entre os dois produtos gerados e análise visual qualitativa.

Primeiramente, buscou-se analisar cada modelo com relação ao objeto real. Para isto, foram medidas distâncias entre os mesmos pontos de referência no objeto real (com a ajuda de um paquímetro) e nos modelos virtuais (utilizando a ferramenta "régua" do software livre Blender - Figura 3.

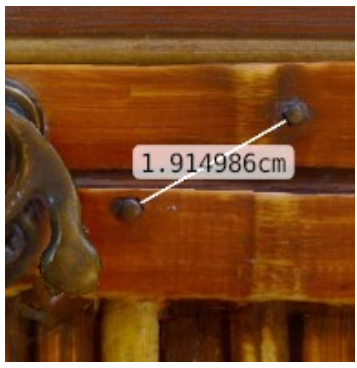

Figura 3. Medição entre pontos no modelo virtual utilizando a ferramenta "régua" do software Blender.

Então, foi testada a confiabilidade das medições lineares. Além disto, os valores das medidas obtidas foram analisados usando a correlação de Pearson [14] e os limites de concordância de $95 \%$ de Bland-Altman [15]. A significância estatística foi estabelecida em 0,05. Após, foi feito o teste t de Student [16] para a diferença entre as medidas real-scanner e real-fotogrametria.
Posteriormente, os modelos 3D foram importados no software livre CloudCompare [17] para serem comparados entre si. Os dois modelos, inicialmente, não estavam perfeitamente sobrepostos devido a diferentes escalas e orientações. Por isto, primeiramente, foram utilizadas as distâncias medidas no objeto real e escalado o objeto virtual, conforme o obtido, georreferenciando os modelos. Em seguida, os dois baús foram alinhados, um sobrepondo o outro, utilizando a ferramenta "Fine registration". Ao final do processo, ambos os modelos ficaram perfeitamente alinhados, apresentando um valor eficaz (RMS) de 0,107. Com a fase de alinhamento, uma única orientação externa absoluta foi então atribuída, mantendo as orientações externas relativas inalteradas para os dois modelos.

Através da ferramenta "Cloud-to-Mesh Distance", foram computadas as distorções do sólido obtido por meio do escaneamento em relação ao da fotogrametria. Para cada vértice do modelo comparado, o ponto mais próximo no modelo referência foi pesquisado, calculando sua distância (Euclidiana) aplicando o "Nível de Octree", ou seja, o nível de subdivisão da Octree em que o cálculo da distância foi realizado.

\section{RESUltados}

Após o processamento, foram obtidos os modelos da Figura 4
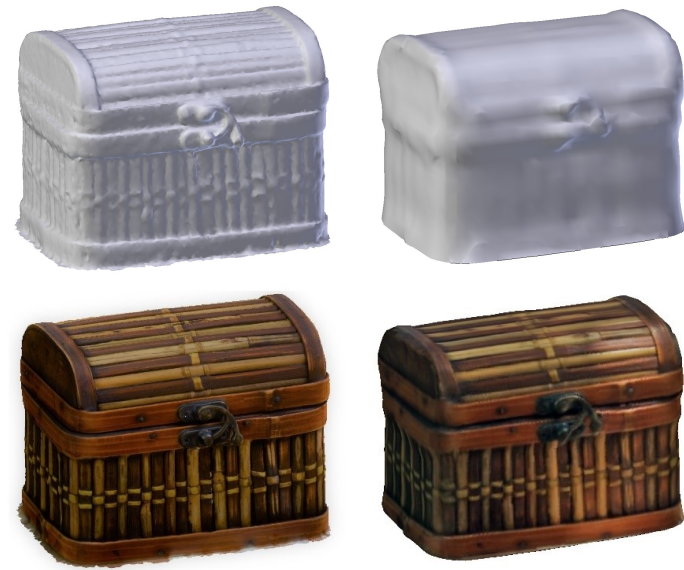

Figura 4. Baús na visão sólido e texturizado - Fotogrametria computadorizada (à esq.); Escaneamento 3D (à dir.).

Algumas informações auxiliares sobre os modelos são apresentadas na Tabela I. São disponibilizados o número final de vértices de cada modelo, o tempo total desde a captura inicial de dados, passando pelo processamento, até a obtenção do resultado final, o tamanho final em $\mathrm{KB}$, a configuração de qualidade (foi utilizada a configuração mais alta no software do scanner e mais baixa no de fotogrametria) e a densidade de vértices por $\mathrm{cm}^{2}$.

Os resultados mostraram uma excelente correlação entre as medidas nos objetos reais comparadas àquelas em objetos virtuais (correlação de Pearson e coeficiente de correlação intraclasse $=0,999$ para objeto real e scanner e 1,000 para objeto real e fotogrametria (IC 95\%)). Enquanto isso, a média 
Tabela I

Algumas CARACTERÍSTICAS DOS MODELOS FINAIS E SUA FABRICAÇÃO.

\begin{tabular}{|c|c|c|c|c|c|}
\hline & Vértices & $\begin{array}{c}\text { Tempo } \\
\text { (min) }\end{array}$ & $\begin{array}{l}\text { Tam. } \\
\text { (KB) }\end{array}$ & $\begin{array}{c}\text { Config. } \\
\text { qual. }\end{array}$ & $\begin{array}{l}\text { Densidade } \\
(\text { vért./cm²) }\end{array}$ \\
\hline Scanner & 3954 & 7 & 518 & Alta & 6,55 \\
\hline Fotogr. & 23136 & 210 & 3890 & Baixa & 33,47 \\
\hline
\end{tabular}

da diferença entre as distâncias real-scanner foi 1,40 e realfotogrametria foi $0,17 \mathrm{~mm}$. O desvio padrão foi 1,99 e 0,38 $\mathrm{mm}$, respectivamente.

Comparações entre as distâncias de cada modelo com o objeto e entre si são apresentadas na Figura 5
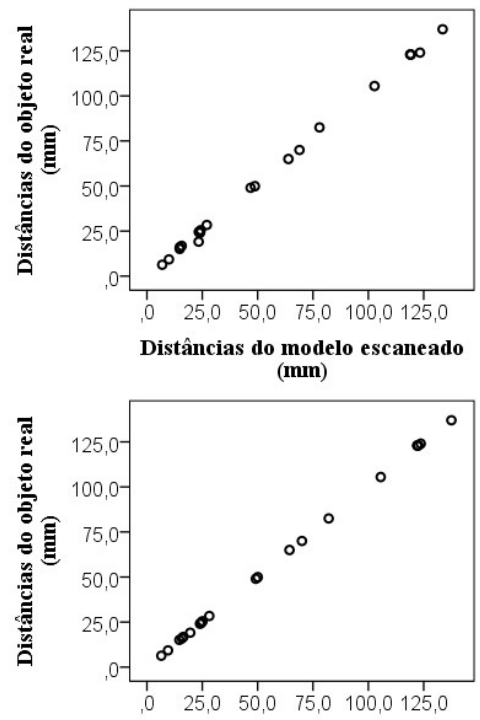

Distâncias do modelo por fotogrametria $(\mathbf{m m})$

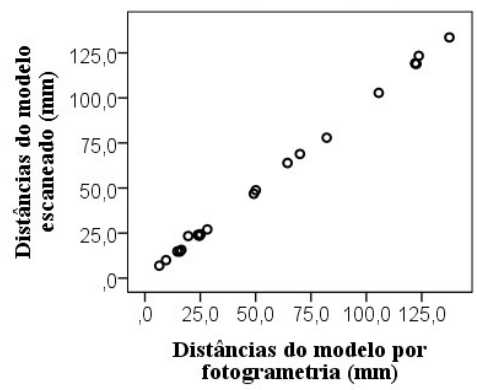

Figura 5. Validação de medidas obtidas nos modelos virtuais 3D em comparação com medições clássicas com paquímetro.

Por meio do teste $t$ de Student, foi identificado que houve uma diferença significativa entre zero e a diferença média das medidas real-scanner, ou seja, que a diferença entre as medidas real-scanner é estatisticamente significante $(p=0,004)$ e que a diferença real-fotogrametria não é estatisticamente significante $(\mathrm{p}=0,059)$.

No gráfico de Bland-Altman (Figura 6, observa-se que não há tendência dos pontos ficarem acima ou abaixo da linha de média entre diferenças $(p=0,218)$, concluindo-se que não houve bias na medição.

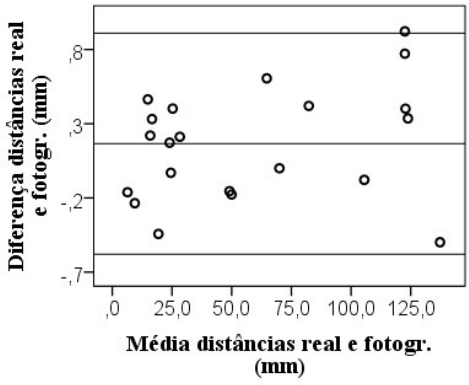

Figura 6. Gráfico de Bland-Altman da diferença entre as distâncias medidas no objeto real e no modelo obtido por fotogrametria.

O processo de análise no CloudCompare gerou quatro campos escalares, um para cada eixo, associados à nuvem de pontos atribuída. Os resultados em termos absolutos são mostrados na Figura 7. A distribuição estatística de Gauss que melhor se adaptou à distribuição efetiva das distâncias foi relacionada ao campo escalar: a operação identificou o valor médio e o desvio padrão.

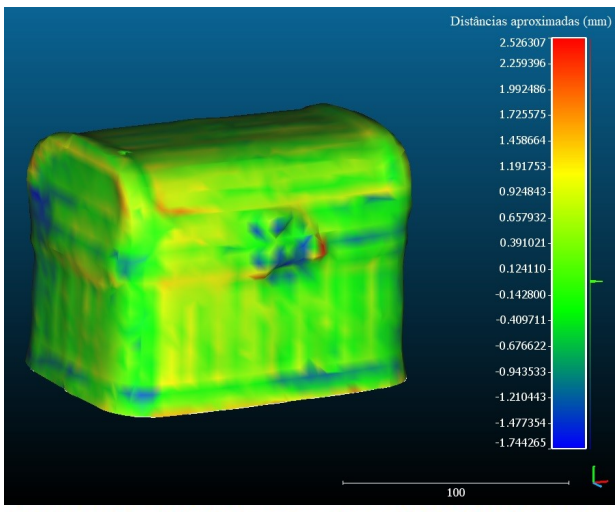

Gauss: média $=0,096764 /$ des. pad. $=0,549901[63$ classes $]$

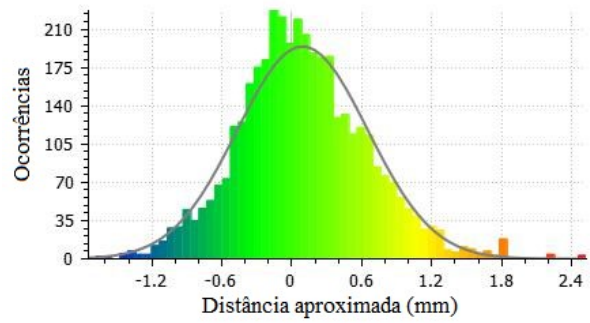

Figura 7. Campo escalar das distâncias entre os vértices do modelo obtido por escaneamento em referência ao modelo obtido por fotogrametria. Os vértices "para fora" estão pigmentados em vermelho, os vértices "para dentro" em azul e os que fazem intersecção entre si em verde. Abaixo, o correspondente histograma demonstrando a distribuição Gaussiana que melhor se adaptou aos valores, incluindo média e desvio padrão. Medidas em milímetros.

A média das distâncias entre vértices dos modelos obtidos por fotogrametria e escaneamento, após sua sobreposição, foi de $0,005838 \mathrm{~mm}$ (desvio padrão $=0,137022 \mathrm{~mm}$ ).

Ademais, fazendo a análise visual, percebe-se que o modelo gerado pelo escaneamento possui distorções na textura e geometria, aparentando estar "derretido" e uma textura com pior qualidade do que o da fotogrametria (ver Figura 8). 


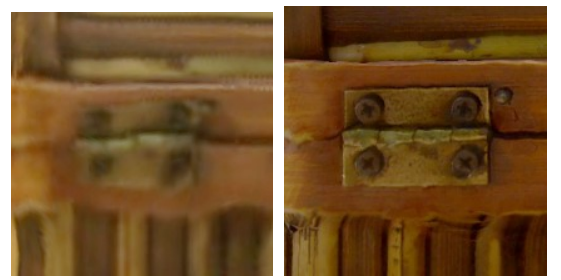

Figura 8. Detalhe visual do modelo obtido por escaneamento (à esq.) e do obtido por fotogrametria (à dir.).

\section{DISCUSSÃO}

Observou-se, primeiramente, que, mesmo na configuração de qualidade máxima do scanner e qualidade mínima do software de fotogrametria, o modelo da primeira técnica teve apenas $17 \%$ do número total de vértices e $13 \%$ do tamanho (KB) do da segunda. Por isto, é natural de se esperar que tenha geometria menos intrincada. Além disto, a textura do mesmo ficou bastante prejudicada, já que o scanner testado possui resolução VGA (640x480 pixels) enquanto as fotos utilizadas na fotogrametria tinham dimensão quase 10 vezes maior.

Analisando a diferença entre medidas do objeto real obtidas com paquímetro e medidas dos objetos virtuais obtidas com a ferramenta "régua" do Blender, observou-se que as distâncias real-fotogrametria foram 8 vezes menores do que real-scanner, e o desvio padrão indicou que a fotogrametria foi mais consistente nessas diferenças.

Mais além, o teste $\mathrm{t}$ de Student indicou um certo nível de concordância entre as medidas real-fotogrametria e que as medidas real-scanner não concordam significativamente entre si.

O teste de sobreposição entre os modelos das duas técnicas mostrou uma média de desvios não importante. A maioria dos valores observados de deslocamento linear estão dentro da faixa de precisão de $95 \%$.

\section{CONClus Ão}

No presente artigo, mostrou-se que é possível fazer um modelo 3D realista utilizando tanto o scanner 3D Structure Sensor quanto por fotogrametria. Estas técnicas foram comparadas por possuírem custos de material similares. Também foram fornecidas informações sobre a confiabilidade dos objetos 3D reconstruídos para medições lineares e para dados de superfície.

Observou-se que o modelo escaneado apresentou maior diferença de distâncias entre seus pontos e do objeto real quando comparado ao modelo obtido por fotogrametria. Mesmo assim, estas diferenças estão na faixa de milímetros.

Adicionalmente, a avaliação qualitativa dos modelos reforça a quantitativa mostrando que a fotogrametria é destacadamente melhor sobre o scanner 3D, gerando um modelo notadamente mais detalhado e preciso tanto em geometria quanto textura em relação ao real, apesar do maior tempo de processamento e menor intuitividade no processo de obtenção do modelo.

Conclui-se que ambas as técnicas podem ser aplicadas a depender do que se deseja obter. Utilizar o scanner 3D possui a grande vantagem da praticidade e rapidez, apresentando muito menor dificuldade em ser utilizado. Portanto, caso seja necessária absoluta precisão na geometria/textura e medidas do modelo, recomenda-se a fotogrametria. Caso apenas necessitese de um esboço, recomenda-se o escaneamento 3D, pela praticidade.

\section{Agradecimentos}

Os autores gostariam de agradecer ao CNPq e à Fapemig pelo financiamento e incentivo para realização deste trabalho, por meio de bolsa PIBIC para as duas primeiras autoras.

\section{REFERÊNCIAS}

[1] J. d. Andrade, "Fotogrametria. sbee," Curitiba. 258p, 1998.

[2] F. Alidoost and H. Arefi, "Comparison of uas-based photogrammetry software for 3d point cloud generation: A survey over a historical site," ISPRS Annals of Photogrammetry, Remote Sensing and Spatial Information Sciences, vol. IV-4/W4, pp. 55-61, 112017.

[3] D. Viggiano, T. Thanassoulas, C. Di-Cesare, G. Cacciola, N. Giorgio, T. Pitsios, and C. Passiatore, "A low-cost system to acquire $3 \mathrm{~d}$ surface data from anatomical samples," vol. 19, pp. 343-349, 012015.

[4] N. J. Groetelaars and A. L. de Amorim, "Tecnologia 3d laser scanning: características, processos e ferramentas para manipulação de nuvens de pontos," in Anais do 15th Iberoamerican Congress of Digital Graphics. Santa Fe, Argentina, pp. 490-494, 2011.

[5] E. Dezen-Kempter, L. Soibelman, M. Chen, and A. V. Müller Filho, "Escaneamento 3d a laser, fotogrametria e modelagem da informação da construção para gestão e operação de edificações históricas," Gestão \& Tecnologia de Projetos, vol. 10, no. 2, pp. 113-124, 2015.

[6] F. Rengier, A. Mehndiratta, H. Von Tengg-Kobligk, C. M. Zechmann, R. Unterhinninghofen, H.-U. Kauczor, and F. L. Giesel, "3d printing based on imaging data: review of medical applications," International journal of computer assisted radiology and surgery, vol. 5, no. 4, pp. 335-341, 2010.

[7] L. Renuncio and C. Loch, "Documentação de patrimônio histórico através da aplicação de técnicas de fotogrametria a curta distância," in Anais do XVIII Congresso Brasileiro de Cartografia. Rio de Janeiro-RJ, pp. 353-355, 1997.

[8] D. Skarlatos and S. Kiparissi, "Comparison of laser scanning, photogrammetry and sfm-mvs pipeline applied in structures and artificial surfaces," ISPRS Annals of the Photogrammetry, Remote Sensing and Spatial Information Sciences, vol. 3, pp. 299-304, 2012.

[9] "Structure Sensor - 3D scanning, augmented reality, and more for mobile devices." Disponível em: https://structure.io/ Acesso em: 26 jun. 2019.

[10] D. Lichti, S. Gordon, M. Stewart, J. Franke, and M. Tsakiri, "Comparison of digital photogrammetry and laser scanning," in Proc. International Society for Photogrammetry and Remote Sensing, pp. 39-44, 2002.

[11] W. Böhler and A. Marbs, "3d scanning and photogrammetry for heritage recording: a comparison," in Proceedings of the 12th International Conference on Geoinformatics, pp. 291-298, Gavle University Press, Sweden, 2004.

[12] “Agisoft Metashape." Disponível em: https://www.agisoft.com/ Acesso em: 26 jun. 2019.

[13] A. Barbasiewicz, T. Widerski, and K. Daliga, "The analysis of the accuracy of spatial models using photogrammetric software: Agisoft photoscan and pix4d," E3S Web of Conferences, vol. 26, p. 00012, 01 2018.

[14] D. Freedman, R. Pisani, and R. Purves, "Statistics (international student edition)," Pisani, R. Purves, 4th edn. WW Norton \& Company, New York, 2007.

[15] J. M. Bland and D. Altman, "Statistical methods for assessing agreement between two methods of clinical measurement," The lancet, vol. 327, no. 8476 , pp. 307-310, 1986.

[16] Student, "The probable error of a mean," Biometrika, pp. 1-25, 1908.

[17] "Cloud Compare - Open Source Project." Disponível em: http://www. cloudcompare.org/ Acesso em: 26 jun. 2019. 